\title{
We Begin with a "Trivial" Condition on Massive Gravitons, and Use That to Examine Nonsingular Starts to Inflation, for GW, with GW Strength and Possible Polarization States?
}

\author{
Andrew Walcott Beckwith \\ Physics Department, College of Physics, Chongqing University, Chongqing, China \\ Email: Rwill9955b@gmail.com, abeckwith@uh.edu
}

How to cite this paper: Beckwith, A.W. (2020) We Begin with a "Trivial" Condition on Massive Gravitons, and Use That to Examine Nonsingular Starts to Inflation, for GW, with GW Strength and Possible Polarization States? Journal of High Energy Physics, Gravitation and Cosmology, 6, 692709.

https://doi.org/10.4236/jhepgc.2020.64048

Received: August 25, 2020

Accepted: October 8, 2020

Published: October 11, 2020

Copyright $\odot 2020$ by author(s) and Scientific Research Publishing Inc. This work is licensed under the Creative Commons Attribution International License (CC BY 4.0).

http://creativecommons.org/licenses/by/4.0/ (c) (i) Open Access

\begin{abstract}
Our Methodology is to construct using a "trivial" solution to massive gravitons, and a nonsingular start for expansion of the universe. Our methodology has many unintended consequences, not the least is a relationship between a small time step, $t$, the minimum scale factor and even the tension or property values of the initial space-time wall, and that is a consequence of a "trivial" solution taking into account "massive" gravitons. I.e. this solution has a mass term times the partial derivative with respect to time of an expression in brackets. The expression in brackets is the cube of a scale factor minus the square of the scale factor. Bonus that this equation is set to zero. It is deemed trivial due to the insistence of having a singular solution. If that is dropped, we have a different venue. In addition, the Friedman equation for nonsingular cosmology can have a quadratic dependence upon a density (of space-time), leading to a way to incorporate right at the surface of the initial "space-time" bubble an uncertainty principle. From there we suggest a first principle Schrodinger equation, with the caveat that time does not exist, within the space-time nonsingular bubble, but is formed right afterwards. From there we again form solutions for strength of GW signals and suggestions as to polarization states. Our quest is motivated by our last articles question, where "We conclude by stating the following question. Can extra dimensions come from a Multiverse feed into Pre-Planckian space-time? See Theorem at the end of this publication. Our answer is in the affirmative, and it has intellectual similarities to George Chapline's work with Black hole physics". From there we next will in future articles postulate conditions for experimental detectors for subsequent data sets to obtain falsifiable data sets.
\end{abstract}




\section{Keywords}

Minimum Scale Factor, Cosmological Constant, Space-Time Bubble, Penrose Singularity

\section{Let Us Now Look at That Famous "Simple Solution" Template for Massive Gravity}

In [1] pages 212-213, we have that there is a Minkowski simple model for massive gravity, leading to

$$
m \cdot \partial_{t}\left(a^{2}-a^{3}\right)=0
$$

Whereas if we us [2], and [3] and really look at [4], we can add in the following as to the scale factor used in Equation (1) which uses at the surface of a presumed non singular start to the expansion of the universe

$$
a(t)=a_{\min } t^{\gamma}
$$

Leading to [4] the inflaton.

$$
\phi=\sqrt{\frac{\gamma}{4 \pi G}} \ln \left\{\sqrt{\frac{8 \pi G V_{0}}{\gamma(3 \gamma-1)}} \cdot t\right\}
$$

And what we will use later the "inflaton potential" we write as

$$
V=V_{0} \cdot\left\{\sqrt{\frac{8 \pi G V_{0}}{\gamma(3 \gamma-1)}} \cdot t\right\}^{\sqrt{\frac{\gamma}{4 \pi G}}-\sqrt{\frac{8 \pi G}{\gamma}}}
$$

Equation (1) and Equation (2) lead us to the interesting restraint of

$$
t=\left(\frac{2}{3 a_{\min }}\right)^{1 / \gamma}
$$

Our preliminary consideration is to have the time, in Equation (5) commensurate with Planck time, whereas a radius of the "bubble" of initial space-time commensurate with Planck length, using the dimensions given in [5].

We will see where this becomes important and how it factors into discussion of the strength of a GW signal and possibly polarization.

\section{A Review of Nonsingular Cosmologies and the Friedman Equation, and How We Utilize Equation (5)}

A given in our work is that within the nucleation of space-time, that time, even as given in Equation (5) simply does not exist, but that we will be able to use the results of Freeze [6] as far as a nonsingular Friedman equation to come up with

$$
H^{2}=\frac{8 \pi}{3 M_{P}^{2}} \cdot\left(\rho-\frac{\rho^{2}}{2|\sigma|}\right)
$$

Here, we have that $\rho$ is a space-time density function, whereas $\sigma$ is re- 
lated to the tension of a space-time bubble presumably of the order of a Planck radius. And we are also using what is given in [7] as far as a Dark Energy model, which we write, for energy density, as given by, if $\mathfrak{R}$ is the Ricci scalar [8] and we use also the notation of [9]. We have also the discussion given in [10] and set $\kappa$ as spacetime curvature, and so then we have

$$
\rho_{D E}=\frac{3 \tilde{\alpha}}{8 \pi} \cdot\left(\dot{H}+2 H^{2}+\frac{\kappa}{a^{2}}\right)=-\frac{\tilde{\alpha}}{8 \pi} \cdot \Re
$$

We cite references [11] [12] and [13] as to the work done with regards to quantum gravity needed to formulate if we can have space-time with a non singular start to expansion of the Universe. We do this because the quantum gravity approximation used, as given in [11] [12] and [13] meant that time as we think of it, at the onset of the bubble of nucleated space-time does not exist in the usual causal structure we identify with, in later expansion of the Universe.

In terms of the bubble of spacetime before inflation, we submit that time does not really exist and that then we will be considering a rewrite of the above as having, effectively $\dot{H}=0$. And the term $\rho_{D E}=\rho$. And if we apply Equation (6), we have within the bubble of spacetime.

Then we have the following, namely

$$
\rho^{2}-\rho \cdot(2|\sigma|)\left(1-\left(\frac{3 \tilde{\alpha}}{8 \pi}\right)^{-1} \cdot\left(\frac{16 \pi}{3 M_{P}^{2}}\right)^{-1}\right)-\left(\frac{16 \pi}{3 M_{P}^{2}}\right)^{-1} \cdot(2|\sigma|) \cdot \frac{\kappa}{a^{2}}=0
$$

Then we get

$$
\begin{aligned}
\rho= & \frac{2|\sigma|}{2} \cdot\left(1-\left(\frac{3 \tilde{\alpha}}{8 \pi}\right)^{-1} \cdot\left(\frac{16 \pi}{3 M_{P}^{2}}\right)^{-1}\right) \\
& \pm \frac{1}{2} \cdot \sqrt{\left(2|\sigma| \cdot\left(1-\left(\frac{3 \tilde{\alpha}}{8 \pi}\right)^{-1} \cdot\left(\frac{16 \pi}{3 M_{P}^{2}}\right)^{-1}\right)\right)^{2}+\left(\frac{16 \pi}{3 M_{P}^{2}}\right)^{-1} \cdot(2|\sigma|) \cdot \frac{8 \kappa}{a^{2}}}
\end{aligned}
$$

This value for $\rho_{D E}=\rho$ at just about the surface of a bubble of initial spacetime nucleation, would be if $\kappa$ were small but not zero

$$
\begin{aligned}
\rho= & \frac{2|\sigma|}{2} \cdot\left(1-\left(\frac{3 \tilde{\alpha}}{8 \pi}\right)^{-1} \cdot\left(\frac{16 \pi}{3 M_{P}^{2}}\right)^{-1}\right) \\
& \cdot\left[1 \pm\left(1+\left(\frac{16 \pi}{3 M_{P}^{2}}\right)^{-1} \cdot \frac{(2|\sigma|) \cdot \frac{4 \kappa}{a^{2}}}{\frac{2|\sigma|}{2} \cdot\left(1-\left(\frac{3 \tilde{\alpha}}{8 \pi}\right)^{-1} \cdot\left(\frac{16 \pi}{3 M_{P}^{2}}\right)^{-1}\right)}\right)\right]
\end{aligned}
$$

We would then be tending toward, if we used

$$
\Delta E \Delta t=\hbar
$$




$$
\Delta t=\frac{l_{\text {Planck }}^{3} \hbar /\left[|\sigma|\left(1-\left(\frac{3 \tilde{\alpha}}{8 \pi}\right)^{-1} \cdot\left(\frac{16 \pi}{3 M_{P}^{2}}\right)^{-1}\right)\right]}{1 \pm \sqrt{1+\left(\frac{16 \pi}{3 M_{P}^{2}}\right)^{-1} \cdot \frac{(2|\sigma|)^{-1} \cdot \frac{8 \kappa}{a^{2}}}{\left(1-\left(\frac{3 \tilde{\alpha}}{8 \pi}\right)^{-1} \cdot\left(\frac{16 \pi}{3 M_{P}^{2}}\right)^{-1}\right)^{2}}}}
$$

If we have, then say small $\kappa$ value

$$
\Delta t \approx \frac{l_{\text {Planck }}^{3} \hbar /\left[|\sigma|\left(1-\left(\frac{3 \tilde{\alpha}}{8 \pi}\right)^{-1} \cdot\left(\frac{16 \pi}{3 M_{P}^{2}}\right)^{-1}\right)\right]}{\left(\frac{16 \pi}{3 M_{P}^{2}}\right)^{-1} \cdot \frac{-(2|\sigma|)^{-1} \cdot \frac{8 \kappa}{a^{2}}}{\left(1-\left(\frac{3 \tilde{\alpha}}{8 \pi}\right)^{-1} \cdot\left(\frac{16 \pi}{3 M_{P}^{2}}\right)^{-1}\right)^{2}}}
$$

Then we would have, say for small $\tilde{\alpha}$ is small, then we have, that we could even see the following, namely

$$
\begin{aligned}
\Delta t & \approx \frac{l_{\text {Planck }}^{3} \hbar /\left[|\sigma|\left(1-\left(\frac{3 \tilde{\alpha}}{8 \pi}\right)^{-1} \cdot\left(\frac{16 \pi}{3 M_{P}^{2}}\right)^{-1}\right)\right]}{\left(\frac{16 \pi}{3 M_{P}^{2}}\right)^{-1} \cdot \frac{-(2|\sigma|)^{-1} \cdot \frac{8 \kappa}{a^{2}}}{\left(1-\left(\frac{3 \tilde{\alpha}}{8 \pi}\right)^{-1} \cdot\left(\frac{16 \pi}{3 M_{P}^{2}}\right)^{-1}\right)^{2}}} \\
& \approx 2 l_{\text {Planck }}^{3} \hbar \cdot\left(-\left(\frac{16 \pi}{3 M_{P}^{2}}\right)+\left(\frac{3 \tilde{\alpha}}{8 \pi}\right)^{-1}\right)
\end{aligned}
$$

In either case, we will be doing our calculations to determine what this has to say as to the frequency of a signal from this event, as well as the strength of GW, and then also the possible polarization states. This would have to be contrasted with Equation (5), as to what that says about bounding values for the input into Equation (15) below.

Let us now refer to a simple but specific model given by Dr. Corda, as to the way one can implement an amplitude for GW [14] which the author views as commensurate with regards to amplitude for this initial system of GW generation at the start of the expansion of the Universe.

From [14] we have that there are minimal extensions of General Relativity for the existence of GW generation which may be thoroughly explored which will enable us to have GW early universe production. Before we get to that we will briefly review what is known about the GW generation of the classical kind which is a precursor to understanding the problems with GW amplitude calculations in the beginning of Space-time evolution. 


\section{Brief Review of the Basics of GW Amplitude Calculations in the Traditional GW Theories}

To do this, we look at [15] which is Maggiore's first volume masterpiece of GW data analysis and collation of data. In doing so, on page 211 , we have that if a $\mathrm{GW}$ were signified to run in the $\mathrm{Z}$ direction, then in lowest order, assuming that we are using, if time is retarded time, then the two main polarizations are describable as

$$
\begin{aligned}
& h_{+}=r^{-1} G c^{-3} \cdot\left(\ddot{M}_{11}-\ddot{M}_{22}\right)=2 r^{-1} G c^{-5} \cdot \int \mathrm{d}^{3} x \cdot \partial_{0}^{2}\left[\left(x_{1}^{2}-x_{2}^{2}\right) \cdot T^{00}\right] \\
& h_{\times}=2 r^{-1} G c^{-3} \cdot\left(\ddot{M}_{12}\right)=2 r^{-1} G c^{-5} \cdot \int \mathrm{d}^{3} x \cdot \partial_{0}^{2}\left[\left(x_{1} x_{2}\right) \cdot T^{00}\right]
\end{aligned}
$$

Here, we have that, usually, $h_{+} \approx h_{x}$.

In doing so, we can write up what we have here with respect to the Einstein Energy tensor as

$$
T^{00} \equiv C \rho_{\text {energy density }}=\frac{\left(R_{00}-g_{00} R\right)}{8 \pi}
$$

Here by [16] we can write

$$
R_{00}=4 h_{+} \ddot{h}_{+}+2 \dot{h}_{+}^{2}+4 h_{\times} \ddot{h}_{x}+2 \dot{h}_{x}^{2}
$$

And the Ricci Scalar as

$$
R \equiv g^{\mu v} R_{\mu v}
$$

Whereas we can then proceed in making sense of what Equation (16), Equation (17) and Equation (18) are saying in terms of Massive Gravity, and relic GW generation.

The action will start in the interpretation of $R_{00}$ Ricci tensor, time domain, and $R$ as a Ricci scalar term.

\section{Interpreting $\boldsymbol{R}_{00}$ Ricci Tensor, Time Domain, and $\boldsymbol{R}$}

[17] gives us a different view of the Equation (16) to Equation (18) terms which we can summarize, below. Afterwards we will go to that procedure brought up by Corda in [14] in terms of relic conditions. Here, the Ricci scalar is given directly as

$$
R=g^{\mu v} R_{\mu v}=6 \Lambda
$$

Whereas the term, given in Equation (17) gets a new paintjob which we can have as by Kolb and Turner [18] for a Roberson-Walker cosmology, if we stick initially with Equation (17) would have the following equality

$$
R_{00}=4 h_{+} \ddot{h}_{+}+2 \dot{h}_{+}^{2}+4 h_{\times} \ddot{h}_{\times}+2 \dot{h}_{\times}^{2} \simeq-3 \frac{\ddot{a}}{a}
$$

Whereas we would re write Equation (19) as the following

$$
R=g^{\mu v} R_{\mu v}=6 \Lambda \approx-6 \cdot\left(\frac{\ddot{a}}{a}+\left(\frac{\dot{a}}{a}\right)^{2}+\frac{2 \kappa}{a^{2}}\right)
$$

In our case we would consider the Equation (17) to be dominant in our problem, and therefore choose Equation (16) to read as 


$$
T^{00} \equiv c \rho_{\text {energy density }}=\frac{\left(R_{00}-g_{00} R\right)}{8 \pi} \approx \frac{c \cdot\left(-3 \frac{\ddot{a}}{a}-g_{00} 6 \Lambda\right)}{8 \pi}
$$

i.e. of all things a negative energy density, which is weird, at the surface of the bubble of space-time, whereas if we use Equation (21) directly we would have, instead

$$
T^{00} \equiv c \rho_{\text {energy density }}=\frac{\left(R_{00}-g_{00} R\right)}{8 \pi} \approx \frac{c \cdot\left(-3 \frac{\ddot{a}}{a}+g_{00} 6\left(\frac{\ddot{a}}{a}+\left(\frac{\dot{a}}{a}\right)^{2}+\frac{2 \kappa}{a^{2}}\right)\right)}{8 \pi}
$$

Whereas then we would have the following to contend with, as far as

$$
h_{+}=\frac{r^{-1} G c^{-4}}{4} \cdot \int \mathrm{d}^{3} x \cdot \partial_{0}^{2}\left[\left(x_{1}^{2}-x_{2}^{2}\right) \cdot \frac{c \cdot\left(-3 \frac{\ddot{a}}{a}-g_{00} 6 \Lambda\right)}{\pi}\right]
$$

Approximated as

$$
h_{+} \approx \frac{r^{-1} G c^{-4}}{4} \cdot \int \mathrm{d}^{3} x \cdot \partial_{0}^{2}\left[\left(x_{1}^{2}-x_{2}^{2}\right) \cdot \frac{c \cdot\left(-3 \frac{\ddot{a}}{a}+g_{00} 6\left(\frac{\ddot{a}}{a}+\left(\frac{\dot{a}}{a}\right)^{2}+\frac{2 \kappa}{a^{2}}\right)\right)}{\pi}\right]
$$

Whereas we could write, the other magnitude as

$$
\begin{gathered}
h_{x}=\frac{r^{-1} G c^{-4}}{4} \cdot \int \mathrm{d}^{3} x \cdot \partial_{0}^{2}\left[\left(x_{1} x_{2}\right) \cdot \frac{c \cdot\left(-3 \frac{\ddot{a}}{a}-g_{00} 6 \Lambda\right)}{\pi}\right] \\
h_{x} \approx \frac{r^{-1} G c^{-4}}{4} \cdot \int \mathrm{d}^{3} x \cdot \partial_{0}^{2}\left[\left(x_{1} x_{2}\right) \cdot \frac{c \cdot\left(-3 \frac{\ddot{a}}{a}+g_{00} 6\left(\frac{\ddot{a}}{a}+\left(\frac{\dot{a}}{a}\right)^{2}+\frac{2 \kappa}{a^{2}}\right)\right)}{\pi}\right]
\end{gathered}
$$

These would have to be configured to be at the surface of the space-time bubble with the Time derivative, in this case, according to Maggiore to be retarded time, and also using Equation (2) for the scale factor. Whereas we could put in different candidates as to the $g_{00}$ term.

\section{Comparing These Values of with the Density Values of Equation (10) for Density, as Put in Would Lead to Equation (24) and Equation (25) Re Written in Terms of the Bubble of Space-Time with a Base of Equation (13) Would Remove the Potential of Negative Values}

Here is the problem if that is done. I.e. the answer would be dependent upon a reading, experimentally upon the tension of the bubble of Space-time presumed. Difficult but not impossible. But necessitating a huge amount of work. $|\sigma|$ would 
need experimental confirmation, whereas we would be using retarded time in the time differentiation.

$$
\begin{aligned}
h_{\times} \approx & 2 r^{-1} G c^{-4} \cdot \int \mathrm{d}^{3} x \cdot \partial_{0}^{2}\left[\left(x_{1} x_{2}\right) \cdot|\sigma| \cdot\left(1-\left(\frac{3 \tilde{\alpha}}{8 \pi}\right)^{-1} \cdot\left(\frac{16 \pi}{3 M_{P}^{2}}\right)^{-1}\right)\right. \\
& \left.\left.\cdot\left[1 \pm\left(1+\left(\frac{16 \pi}{3 M_{P}^{2}}\right)^{-1} \cdot \frac{(2|\sigma|) \cdot \frac{4 \kappa}{a^{2}}}{\frac{2|\sigma|}{2} \cdot\left(1-\left(\frac{3 \tilde{\alpha}}{8 \pi}\right)^{-1} \cdot\left(\frac{16 \pi}{3 M_{P}^{2}}\right)^{-1}\right)}\right)\right]\right]\right]
\end{aligned}
$$

And

$$
\begin{aligned}
h_{+} \approx 2 r^{-1} G c^{-4} \cdot \int \mathrm{d}^{3} x \cdot \partial_{0}^{2}\left[\left(x_{1}^{2}-x_{2}^{2}\right) \cdot|\sigma| \cdot\left(1-\left(\frac{3 \tilde{\alpha}}{8 \pi}\right)^{-1} \cdot\left(\frac{16 \pi}{3 M_{P}^{2}}\right)^{-1}\right)\right. \\
\left.\cdot\left[1 \pm\left(1+\left(\frac{16 \pi}{3 M_{P}^{2}}\right)^{-1} \cdot \frac{(2|\sigma|) \cdot \frac{4 \kappa}{a^{2}}}{\frac{2|\sigma|}{2} \cdot\left(1-\left(\frac{3 \tilde{\alpha}}{8 \pi}\right)^{-1} \cdot\left(\frac{16 \pi}{3 M_{P}^{2}}\right)^{-1}\right)}\right)\right]\right]
\end{aligned}
$$

This though lacks the third term from an additional polarization term which was used by Corda in [14] which will be referred to later. Before doing that, look at the additional development we could do, with Equation (10) for a GW density term. We can though add in the cross polarization term before going to the Corda treatment in [14]. It is his Equation (48). Which is our Equation (28)

$$
h_{\mu v}=A^{+}(t-z) e_{u v}^{+}+A^{\times}(t-z) e_{u v}^{\times}+h_{m}\left(t-v_{G} z\right)
$$

\section{Linking to the Corda Approach in [14] for Relic GW Conditions, i.e. Adding Equations Together}

The Corda approach, in Reference [14] is in its Equation (48) a representation we will refer to as setting, if we used the cosmological parameter referenced in Equation.

Here we make the following identification, namely

$$
\begin{aligned}
A^{+}(t-z) e_{u v}^{+} & \leftrightarrow h_{+} \\
A^{\times}(t-z) e_{u v}^{\times} & \leftrightarrow h_{x}
\end{aligned}
$$

The missing term is in $h_{m}\left(t-v_{G} z\right)$. We will make some comments as to this additional term. 


\section{Adding in $h_{m}\left(t-v_{G} z\right)$ and Coming up with a Primordial} GW Amplitude Generation

To do this we will consider having a look at the Equation (45) of reference [14] which we will write as

$$
\mathrm{d} \rho_{G W}(\text { relic GW }) \approx \hbar \cdot \frac{\left(H_{0}^{2} H_{d S}^{2}\right)}{4 \pi^{2} c^{3}} \cdot \frac{\mathrm{d} \omega}{\omega}
$$

We claim that this would be commensurate with the Hubble expansion $H_{0}$ defined by setting it to today's space time crucial density

$$
H_{0}^{2} \text { (Today) } \equiv \frac{8 \pi G \rho_{\text {critial density }}}{3 c^{2}}
$$

Whereas earlier we would have

$$
H_{d S}^{2} \text { (Beginning) } \equiv \frac{8 \pi G \rho_{d S}}{3 c^{2}}
$$

Our approximation in our paper is to use the following substitution, namely make the following substitution

$$
\begin{aligned}
& c \rho_{d S}=\left.\left.\left.\frac{\left(R_{00}-g_{00} R\right)}{8 \pi}\right|_{d S}\right|_{d S} \approx \frac{\left(-3 \frac{\ddot{a}}{a}-g_{00} 6 \Lambda\right)}{8 \pi}\right|_{d S}
\end{aligned}
$$

Then to use an integrated version of this in Equation (30), Equation (31), and Equation (32) and then come up with a linkage to analyzing

$$
\rho_{G W}(\text { relic GW }) \approx \hbar \cdot \int \frac{\mathrm{d} \omega}{\omega} \frac{\left(H_{0}^{2} H_{d S}^{2}\right)}{4 \pi^{2} c^{3}}
$$

Re write this as follows set

$$
\left.H_{d S}^{2} \equiv \frac{8 \pi G \rho_{d S}}{3 c^{2}} \approx \frac{8 \pi G}{3 c^{2}} \cdot \frac{\left(-3 \frac{\ddot{a}}{a}+g_{00} 6\left(\frac{\ddot{a}}{a}+\left(\frac{\dot{a}}{a}\right)^{2}+\frac{2 \kappa}{a^{2}}\right)\right)}{8 \pi}\right|_{d S}
$$

Then the relic GW density goes as

$$
\begin{aligned}
\rho_{\mathrm{GW} \mathrm{relic}} & \approx \hbar \cdot \int \frac{\mathrm{d} \omega}{\omega} \frac{\left(H_{0}^{2} 2 G \rho_{d S}\right)}{3 \pi c^{5}} \\
& \approx \frac{\hbar H_{0}^{2} G}{c^{5}} \cdot \int \frac{\mathrm{d} \omega}{\omega} \cdot\left\{\left.\frac{\left(-3 \frac{\ddot{a}}{a}+g_{00} 6\left(\frac{\ddot{a}}{a}+\left(\frac{\dot{a}}{a}\right)^{2}+\frac{2 \kappa}{a^{2}}\right)\right)}{12 \pi^{3}}\right|_{d S}\right\}
\end{aligned}
$$

This would lead to a third relic condition GW contribution we would call 


$$
h_{m}\left(t-v_{G} z\right) \approx 2 r^{-1} G c^{-4} \cdot \int \mathrm{d}^{3} x \cdot \partial_{0}^{2}\left[\left(x_{1} x_{2}\right) \cdot \rho_{\mathrm{GW} \text { relic }}\right]
$$

\section{What about Tensor-Mode, Vector-Mode and Scalar-Mode Gravitons as Spin Zero and Spin 1 "Gravitons"?}

We will review our work further but it will be in strict fidelity with what Wen Hao and Fangyu Li did, which is in ARXIV and was just recently published in European Journal C, i.e. see this [19].

Reference [19] is unusually complete. What the author is arguing for is that since the details of the scalar and vector mode interactions are highly detector specific, that adding in these 2 additional polarization states, akin to the five polarization states of massive gravitons will await fine tuning the details of the material in this document, as to the first three polarizations, as well as getting more details as to the instrumentation of the Li Baker detector. This will be followed up upon, pending utilizing the details as to the following document [20] [21].

My guess is that what is done in [19] is for higher than four dimensional, brane physics models, whereas our starting point is for $3+1$ dimensional models, but that the differences are due strictly to higher dimensions employed.

Not that this is alluded to, as far as future work, we will go to the issue of relic GW frequencies.

\section{Order of Magnitude Estimates for Frequency, Say of Relic GW}

We use the most direct route toward doing it, and say if we used

$$
\omega \approx \frac{\left(1 \pm \sqrt{1+\left(\frac{16 \pi}{3 M_{P}^{2}}\right)^{-1} \cdot \frac{(2|\sigma|)^{-1} \cdot \frac{8 \kappa}{a^{2}}}{\left(1-\left(\frac{3 \tilde{\alpha}}{8 \pi}\right)^{-1} \cdot\left(\frac{16 \pi}{3 M_{P}^{2}}\right)^{-1}\right)^{2}}}\right)}{l_{\text {Planck }}^{3} \hbar /\left[|\sigma|\left(1-\left(\frac{3 \tilde{\alpha}}{8 \pi}\right)^{-1} \cdot\left(\frac{16 \pi}{3 M_{P}^{2}}\right)^{-1}\right)\right]}
$$

For sufficiently large $\tilde{\alpha}$ value we hope we have a negative $\kappa$ value

$$
\omega \approx \frac{8 \kappa}{a^{2}} /\left[2 l_{\text {Planck }}^{3} \hbar \cdot\left(-\left(\frac{16 \pi}{3 M_{P}^{2}}\right)+\left(\frac{3 \tilde{\alpha}}{8 \pi}\right)^{-1}\right)\right]
$$

If instead we have a small $\tilde{\alpha}$ value

$$
\omega \approx \frac{8 \kappa}{a^{2}} /\left[2 l_{\text {Planck }}^{3} \hbar \cdot\left(+\left(\frac{3 \tilde{\alpha}}{8 \pi}\right)^{-1}-\left(\frac{16 \pi}{3 M_{P}^{2}}\right)\right)\right]
$$

These expressions should be compared to Equation (5) for which we can write, say [14]

$$
\Delta E \Delta t \approx \hbar \equiv \hbar \omega \Delta t \approx \hbar \omega \cdot\left(\frac{2}{3 a_{\min }}\right)^{1 / \gamma} \Rightarrow \omega \approx \hbar^{-1} \cdot\left(\frac{2}{3 a_{\min }}\right)^{-1 / \gamma}
$$


We think that this means that at the boundary of a space time bubble, that this would force us to have an enormous value for $\gamma$. We would also, if the initial energy were just at the boundary of the bubble of space time, have the odd situation for which we would have the following, namely at the surface of the bubble, so if one used Planck units with $l_{\text {Planck }} \equiv \hbar \equiv G \equiv 1$ we could have the following weird situation

$$
\begin{aligned}
& V=V_{0} \cdot\left\{\sqrt{\frac{8 \pi G V_{0}}{\gamma(3 \gamma-1)}} \cdot t\right\}^{\sqrt{\frac{\gamma}{4 \pi G}}-\sqrt{\frac{8 \pi G}{\gamma}}} \\
& \underset{\text { lplanck }^{\prime}=\hbar \equiv=1}{\longrightarrow} V_{0} \cdot\left\{\sqrt{\frac{8 \pi V_{0}}{\gamma(3 \gamma-1)}} \cdot t\right\}^{\sqrt{\frac{\gamma}{4 \pi}}-\sqrt{\frac{8 \pi}{\gamma}}} \approx \omega \propto\left(\frac{2}{3 a_{\text {min }}}\right)^{-1 / \gamma}
\end{aligned}
$$

To put it mildly, we would need a lot more experimental data sets! All this leads to considerable interplay between the equations given in Equation (38) to Equation (42). We will discuss this in our conclusions. Keep in mind that the interplay with all of this, we will try to right after the Bubble of initially nucleated Space-time to have a linkage to simpler Quantum mechanics involved as seen in [22]. In addition we wish to in doing so to have fidelity with the insights of Christian Corda, as seen in [23] as to mixed Scalar-Tensor modes and $f(R)$ models of Gravity.

In our write up of Equation (38) to Equation (42) we are assuming for all general purposes that the scale factor would have a minimum value. Keep in mind that the surface of the bubble would be, in fidelity with reference [3] be obeying having the scale factor at a minimum value and that in line with inflationary $\mathrm{e}$ folds of 60 [15] [24], or 1.14 times $10^{26}$. If this is kept in mind, and say that we have a frequency range of about $10^{37} \mathrm{Hertz}$, as a result of the above, we would then have say $10^{11}$ Hertz for Earth signals taken for GW detection.

\section{What Is the Strength of a Signal for Our Model?}

GW signals have a simple strength of GW moniker [24] [25] The simplest idea is to look at the behavior of GW from a massive black hole, and in doing so, the strain is simply $M$ the mass of a black hole, $r=$ distance of source to Earth from Black hole, with $v$ as given in Equation (43) being the speed of masses, just short of the speed of light. Then

$$
h \approx \frac{G M}{c^{2}} \cdot \frac{1}{r} \cdot\left(\frac{v}{c}\right)^{2}
$$

If the original universe were acting say as a black hole just before the onset of the black hole exploding via the violence of inflation, if we wish to have an observed GW signal, and have say $v / c$ about 1 , in terms of the early universe, with $r$ being the distance of the expansion in 13.6 billion years, we could have a configuration for which if we were near the initial start point of the Universe, that Equation (43) would be of the order of 1, whereas, the e fold expansion of $10^{26}$ from an initial start point would then have say a signal strength of the order of 
$10^{-25}$ to $10^{-26}$, and if so a strain value of $10^{-26}$ would be commensurate with GW signal detectors being designed as of Earth orbit where we are right now. Verifying though the initial configuration of Equation (43) as approaching unity, though, would require serious investigative work and would entail perhaps spending attention to the fractal geometry given in [17] [26].

The entire reference in [26] is essentially a referencing of the need for fractal dimensionality, and where it fits in, as far as the geometry of space-time. On page 94 of [26] the Hausdorff-Besicovitch dimension, which is fractal is done, in gory detail. Why this is important? Note that in [3] we made reference to [28], which if $d$ (dimension) is fractal, means that the temperature for the universe, so assumed, has many surprises if we wish to connect Equation (44) with a scaling of $\hbar \omega$ i.e. there is then likely not a simply linear relationship between temperature, and frequency, hence we have to be more careful.

$$
\Delta E \equiv \frac{d(\operatorname{dim}) k_{B} T_{\text {temperature universe }}}{2}
$$

Now, make use of [3] again and this relationship below though will stand the test of time, but it is NOT dependent upon frequency! Here $N_{g}$ is a count of gravitons which may commence from our earlier generation of early universe GW. From [3]

$$
\begin{aligned}
& {\left[N_{g} / \text { Volume }(\text { Planck units })\right] \sim S(\text { entropy density })} \\
& \sim\left(\frac{2 \pi^{2} g^{*}}{45}\right) \cdot\left(T_{\text {Universe }} / T_{\text {Planck }}\right)^{2} \\
& \Rightarrow\left(T_{\text {Universe }} / T_{\text {Planck }}\right)^{2} \approx\left(\frac{2 \pi^{2} g^{*}}{45}\right)^{-1} \cdot\left[N_{g} / \text { Volume }(\text { Planck units })\right]
\end{aligned}
$$

In other words, due to fractal geometry, while Equation (22) and Equation (23) may hold, as stated in [3], we may have some observational surprises as far as frequency spectrum data sets as far as GW from relic initial GW generating events in the early universe.

In other wise, while the scaling of $10^{-26}$ downward in terms of general frequency from a nonsingular universe starting point is likely correct, the details of the frequency spectrum in the modern era from Primordial beginnings may be a bit different from what we think.

\section{Is There Another Way to Form a Quantum Wavefunction of the Universe Rather than Wheeler De Witt?}

In [27] there is reference to a solution for which we still have

$$
H(\text { Hamiltonian }) \cdot \Psi(\text { wavefunction universe })=0
$$

Whereas we have, $\kappa=\mp 1,0$ and

$$
H(\text { Hamiltonian })=\left(\frac{3 \pi c^{2}}{4 G}\right) \cdot a^{2} \cdot\left(\frac{4 G^{2} P_{a}^{2}}{9 \pi^{2} c^{4} a^{4}}+\frac{\kappa c}{a^{2}}-\frac{8 \pi G \rho}{3 c^{2}}\right)
$$


Whereas [27] sets $\rho$ as an energy density, for a whole slew of grab bag assorted "topics", and

$$
P_{a}^{2} \rightarrow \frac{\hbar}{a^{\breve{P}^{*}}} \cdot \partial_{a} \cdot \partial_{a}
$$

Here, $\breve{p} *$ is a so called Order parameter, and after a small scale factor solution we would get a linear combination of Bessel and Hankel functions for the wavefunction of the universe, which for small scale factor allowed to go to zero we have, if $c_{1}$ and $c_{2}$ are constants, and the scale factor can get arbitrarily small. The Equation (49) below is specified by Reference [27].

$$
\Psi \text { (universe) } \underset{a \rightarrow 0}{\longrightarrow} c_{1}+c_{2} a^{1-\hat{p}^{*}}
$$

Keep in mind that when we do this we are using the time dependence given by Equation (37), Equation (38) and Equation (39) whereas we will be specifying a frequency dependence given by Equation (40).

So, how could we write in a minimum time step whereas we also have a quantum function?

I will make a suggestion as such, whereas, it may be not accepted. But this is my candidate. From Gasiorowicz [14], for a wave function just outside the minimum scale factor, I would try using Equation (39) and Equation (49) to come up with a wavefunction at the surface of the space-time bubble which would have much the same information as given in Equation (27) above. To do this though we will refer to a result by Shestakova [28], and [29], and then re interpret it via some physics from Stephen Gasiorowicz [14] and compare that directly with Equation (46), and Equation (47).

\section{Simple QM Wavefunction at the Surface of the Space-Time Non Singular Start Point Matched against Equation (27)}

We begin with [28] and [29] by T. P. Shestakova where we have on its page 9, if we use order parameter $p=1$, the following S.E. and also a solution, which is dependent upon a scale factor with $\tilde{\lambda}^{*}$ a dimensional factor put in as a fudge factor

$$
\frac{\mathrm{d}^{2} \Psi_{\text {specialized }}}{\mathrm{d} a^{2}}+a^{-1} \frac{\mathrm{d} \Psi_{\text {specialized }}}{\mathrm{d} a}+a^{2} \tilde{\lambda}^{*} \Psi_{\text {specialized }}=0
$$

If so then, we will be looking at, using Arfken, $4^{\text {th }}$ edition, page 667 [30] so that the solution to Equation (50), given by, [28] and [29] has the form

$$
\Psi_{\text {specialized }}=\tilde{C} K_{0}\left(\frac{\tilde{\lambda}^{*} a^{2}}{2}\right) \approx \tilde{C} \cdot\left\{-\ln \left(\frac{\tilde{\lambda}^{*} a^{2}}{2}\right)-\tilde{\gamma}+\ln 2\right\}
$$

Note that the CRC handbook, [31] gives us an expansion so we can do the following 


$$
\begin{aligned}
\Psi_{\text {specialized }} & =\tilde{C} K_{0}\left(\frac{\tilde{\lambda}^{*} a^{2}}{2}\right) \\
& \approx \tilde{C} \cdot\left\{\left(1-\left(\frac{\tilde{\lambda}^{*} a^{2}}{2}\right)\right)-\frac{1}{2}\left(1-\left(\frac{\tilde{\lambda}^{*} a^{2}}{2}\right)\right)^{2}+\cdots-\tilde{\gamma}+\ln 2\right\}
\end{aligned}
$$

Here if $a=a_{\min }$ is very small whereas we take $\tilde{\lambda}^{*} \rightarrow 1$ we would have

$$
\Psi_{\text {specialized }}=\tilde{C} K_{0}\left(\frac{\tilde{\lambda}^{*} a^{2}}{2}\right) \underset{\tilde{\lambda}^{*} \rightarrow 1, a \rightarrow a_{\min }}{\longrightarrow} \tilde{C} \cdot\left\{\left(1-\left(\frac{\tilde{\lambda}^{*} a_{\min }^{8}}{2}\right)\right)+\cdots-\tilde{\gamma}+\ln 2\right\}
$$

Leading to a comparison between two forms of the Wheeler De Witt equation give the same information, i.e. questioning if the following are equivalent

$$
\Psi_{\text {specialized }} \tilde{C} \cdot\left\{\left(1-\left(\frac{\tilde{\lambda}^{*} a_{\min }^{8}}{2}\right)\right)+\cdots-\tilde{\gamma}+\ln 2\right\} \rightleftarrows\left(c_{1}+c_{2} a_{\min }^{1-\tilde{\tilde{p}}^{*}}\right)(?)
$$

If so, and the answer is yes then we will then have to address what a minimum bubble of space-time ascertains as to future developments of space-time, and for this we will be looking at [14] for inspiration.

\section{How to Link Equation (54) to a Schrodinger Type Equation for Planck Time Physics}

The relationship can be ascertained as to stating that we can use right at the surface of the space-time bubble

$$
\begin{aligned}
& \Delta E \Delta t \approx \hbar \equiv \hbar \omega \Delta t \approx \hbar \omega \cdot\left(\frac{2}{3 a_{\min }}\right)^{1 / \gamma} \Rightarrow \omega \approx \hbar^{-1} \cdot\left(\frac{2}{3 a_{\text {min }}}\right)^{-1 / \gamma} \\
& \therefore \Delta E \propto E_{\min } \propto\left(\frac{2}{3 a_{\text {min }}}\right)^{-1 / \gamma} \\
& \therefore \Psi_{\text {Schrodinger equation }}(\text { surface space time bubble }) \\
& \approx \text { value } \frac{}{l_{\text {Planck }=\hbar=G=1}} \exp \left(i\left(\frac{2}{3 a_{\text {min }}}\right)^{-1 / \gamma} \cdot t_{\text {min }}\right)
\end{aligned}
$$

Keep in mind that this is dependent upon a linkage to Equation (34), which has no explicit time dependence whereas Equation (55) has a lot of time dependence.

\section{And What about Polarization States, Especially If We Have Massive Gravitons?}

See [32]. Our supposition is that as given in [32] that the massive gravity signature we would seek would be commensurate with "pure longitudinal and transverse breathing polarizations in the massive Horndeski theory and $f(R)$ gravity". The details of such are given in their [32] document as given just above their Equation (34) whereas we would have us calculate the electric component $R$ (tjtk) of the Riemann tensor, as given in their document on page 10 of their arXIV document. 
The details of their Equation (34) as in Reference [32] would in the end have to be made fiducially relevant to the experimental platform as selected by an experimental gravity research team, and in itself would require a massive summary of known and sought after we have already outlined a procedure tying in what we did with [14] as given by Dr. Corda. We intend to find Graviscalar and Gravivecor contributions, in line with work presented which is in fidelity with quantum gravity states as summarized already in this document.

\section{Summing Up: And a Path Forward}

1) I am expecting a strain of Gravitational wave strength of $h \sim 10^{-25}$ to $10^{-26}$.

2) A frequency range for GW detected in Earth orbit of about $10^{10} \mathrm{Hertz}$, which could be commensurate with initial GW of $10^{36}$ to $10^{37}$ Hertz for reasons which are in this manuscript. There would be due to e fold values of 60 a dramatic drop in frequencies.

3) The polarization states to watch, indicating if possible, would be for massive gravitons.

4) Should there be a linkage between an initial worm hole style start to the formation of the observable universe, as a compliment to the big bank/inflation metrics, the author strongly recommends a review of the article given by Bruno J. Barros and Francisco S. N. Lobo as to relevant Wormhole geometry [33], and the question of negative energy states in a wormhole threat. If as may be the case in nonsingular starts to the expansion of the universe, negative energy states make an appearance, this may be a clue as to what is called $f(R)$ geometries which in turn may have relevance to some of the polarization states in [32].

The wormhole analogy may be crucial as to looking at the linkage between super massive black holes and recycling of the universe, which may be investigated later.

5) Also the author recommends a doubling down on the physics of this diagram in the last [3] article.

See Figure 1 below which is in [3]. We argue that the initial physics, may help us understand more of the self replication phenomena so outlined.

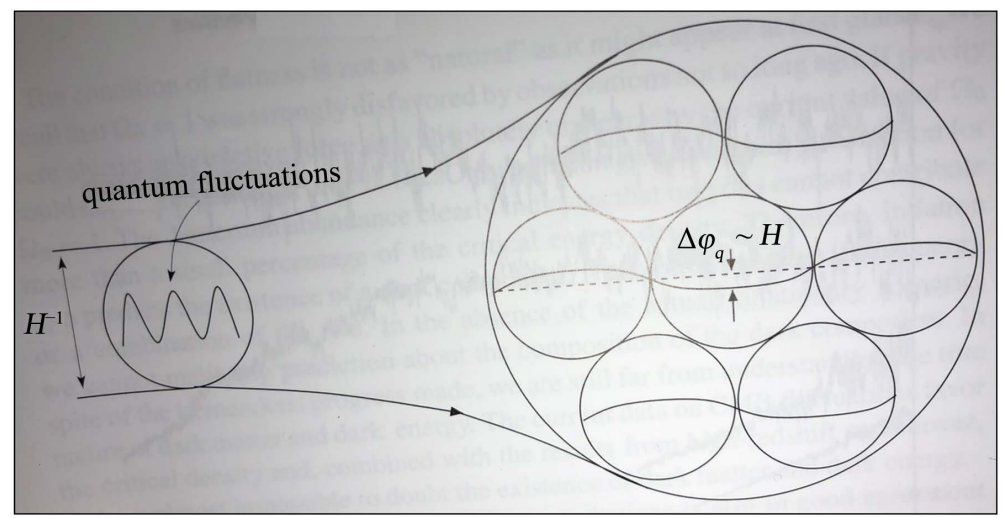

Figure 1. Which is from reference [3]. 
We also should understand if the physics of a linkage between Equation (33) and Equation (34) play a role as well.

6) Finally, and not least is to see if our feed into Equation (33) to Equation (34) and linkages to Figure 1, have in their genesis, the methodologies discussed in [34], i.e. as brought up in Phase transitions and the renormalization group as brought up by Jean-Zinn-Justin, which has a very interesting discussion of the Nancy Kerrigan problem, i.e. as of page 133 of [34] which states that DE did NOT interfere with, or put constraints upon structure formation. We submit that Figure 1 is a case of the DE density, initially being replaced as we did earlier, in the manuscript with cosmological energy density, using the equations we copied from Gong, et al. [32]. This requires further investigation, and may if resolved have a tie into Figure 1 physics, of [3].

7) Doing all the above, would be hard, but would be the cornerstone of introducing the concept of using the construction of a non-Archimedean field to physics [35], which would be a way also to take into account what may be fractal geometry incorporated into space-time physics. In mathematics, a non-Archimedean ordered field is an ordered field that does not satisfy the Archimedean property. Examples are the Levi-Civita field, the hyperreal numbers, the surreal numbers, and the field of rational functions with real coefficients with a suitable order.

This would be a way to understand more of Equation (22), if the dimensional coefficient, $d$, is rep-resenting fractal geometry.

\section{And Now for Some Very Specific Goals for the Future}

We have referenced several different polarization methods for massive gravitons. Our idea is to form a bridge between our extension between [14] as a minimal extension of relativity and the methods brought up by Dr. Hao Wen, Dr. Fangyu $\mathrm{Li}$, and others recently as published in European Physics Journal C, given by [19] with an eye toward coming up with specific counter parts to the work of Gong, for massive gravitons [32]. The work in [19] is outstanding but in a sense very general, as we will try to look at what polarization states survive the onset of quantum gravity conditions at our nonsingular start to the universe.

In addition, the author at the $4^{\text {th }}$ Zeldovich conference, November 11 , at 14:00 CET time made reference to the enduring mystery of error bars in the CMBR measurement as discussed by Abhay Akshenkar [36] of how his loop quantum gravity may form a solution to this problem. My entire nonsingular start to the evolution of the universe was initiated as a way to attack this problem addressed by Abhay and I will endeavor to do more on that in a future publication.

Keep in mind that there are 61 or so e folds from the start to the finish of inflation, allowing the universe to expand to the size of a large grapefruit, and that the total amount of expansion in 13.4 billion years of evolution is about $141 \mathrm{e}$ folds.

Finally Karen Freeze in Zeldovich 4 [37] spoke on the physics of massive Dark Stars which she said may contribute to supermassive black hole formation in the center of elliptical galaxies. This insight will be part of an extension of our work into gravitational astronomy and will be referenced as well in a future publication. 


\section{Acknowledgements}

This work is supported in part by National Nature Science Foundation of China grant No. 11375279.

Personal big thank you to Dr. Christian Corda for his outstanding questions which are highlighted in this manuscript.

\section{Conflicts of Interest}

The author declares no conflicts of interest regarding the publication of this paper.

\section{References}

[1] Tolley, A.J. (2015) Cosmological Applications of Massive Gravity. In: Papantonopoulos, E., Ed., Modifications of Einstein's Theory of Gravity at Large Distances. Lecture Notes in Physics, Springer, Cham, vol. 892, 203-224. https://doi.org/10.1007/978-3-319-10070-8_8

[2] Amico, G.D., de Rham, C., Dubosvsky, S., Gabadadze, G., Pirtskhalava, D. and Tolley, A.J. (2011) Massive Cosmologies. Physical Review D, 84, 124046. https://arxiv.org/abs/1108.5231 https://doi.org/10.1103/PhysRevD.84.124046

[3] Beckwith, A. (2020) How to Obtain a Mass of a Graviton, and Does This Methodology Lead to Voids? Journal of High Energy Physics, Gravitation and Cosmology, 6, 416-439. https://doi.org/10.4236/jhepgc.2020.63032

[4] Padmanabhan, T. (2006) An Invitation to Astrophysics. World Press Scientific, World Scientific Series in Astronomy and Astrophysics: Volume 8. World Scientific Publishing Co. Pte Ltd., Singapore. https://doi.org/10.1142/6010

[5] Wesson, P.S. (1980) The Application of Dimensional Analysis to Cosmology. Space Science Reviews, 27, 109-153. https://doi.org/10.1007/BF00212237

[6] Freeze, K., Brown, M. and Kinney, W. (2012) The Phantom Bounce: A New Proposal for an Oscillating Cosmology. In: Mersini-Houghton, L. and Vaas, R. Eds., The Arrows of Time. Fundamental Theories of Physics, Springer, Berlin, Heidelberg, vol. 172, 149-156. https://doi.org/10.1007/978-3-642-23259-6_7

[7] Li, M., Li, X.-D., Wang, S. and Wang, Y. (2015) Dark Energy. Peking University Press, World Scientific, Singapore, Republic of Singapore. https://doi.org/10.1142/9293

[8] Ahmedov, H. and Aliev, A.N. (2011) More on New Massive Gravity: Exact Solutions. Physical Review Letters, 106, 021301. https://doi.org/10.1103/PhysRevLett.106.021301

[9] Misner, C., Thorne, K.S. and Wheeler, J.A. (1973) Gravitation. W. H. Freeman, Princeton University Press, San Francisco.

[10] Dray, T. (2015) Differential Forms and the Geometry of General Relativity. CRC Press, Boca Raton, Florida. https://doi.org/10.1201/b17620

[11] Rovelli, C. (2001) Notes for a Brief History of Quantum Gravity. The 9th Marcel Grossmann Meeting,Rome, Italy, 2-8 July 2000, 1-32.

[12] Hamber, H.W. and Williams, R.M. (2011) Discrete Wheeler-DeWitt Equation. Physical Review D, 84, 104033. https://doi.org/10.1103/PhysRevD.84.104033

[13] Hamber, H.W., Toriumi, R. and Williams, R.M. (2012) Wheeler-DeWitt Equation in $2+1$ Dimensions. Physical Review D, 86, 084010 . 
https://doi.org/10.1103/PhysRevD.86.084010

[14] Corda, C. (2008) Primordial Production of Massive Relic Gravitational Waves from a Weak Modification of General Relativity. Astroparticle Physics, 30, 209-215. https://arxiv.org/abs/0812.0483 https://doi.org/10.1016/j.astropartphys.2008.09.003

[15] Maggiorie, M. (2008) Gravitational Waves, Volume 1, Theory and Experiment. Oxford University Press, New York City, New York, USA.

[16] http://www.tapir.caltech.edu/ chirata/ph236/2011-12/lec14.pdf

[17] Tasinato, G., Koyama, K. and Niz, G. (2013) Exact Solutions in Massive Gravity. Classical and Quantum Gravity, 30, 184002. https://doi.org/10.1088/0264-9381/30/18/184002

[18] Kolb, E. and Turner, M. (1990) The Early Universe, Frontiers in Physics, Lecture Note Series, Volume 69. Addison-Wesley Publishing Company, Menlo Park, California.

[19] Li, F.-Y., Wen, H., Fang, Z.-Y., Li, D. and Zhang, T.-J. (2018) Electromagnetic Counterparts of High-Frequency Gravitational Waves Having Additional Polarization States: Distinguishing and Probing Tensor-Mode, Vector-Mode and Scalar-Mode Gravitons. https://arxiv.org/pdf/1712.00766.pdf

[20] Li, F.Y., Baker Jr., R.M.L., Fang, Z.Y., Stepheson, G.V. and Chen, Z.Y. (2008) Perturbative Photon Fluxes Generated by High-Frequency Gravitational Waves and Their Physical Effects. The European Physical Journal C, 56, 407-423. https://doi.org/10.1140/epjc/s10052-008-0656-9

[21] Li, F.Y., et al. (2009) Signal Photon Flux and Background Noise in a Coupling Electromagnetic Detecting System for High Frequency Gravitational Waves. Physical Review D, 86, 064013. https://doi.org/10.1103/PhysRevD.80.064013

[22] Gasiorowicz, S. (1966) Elementary Particle Physics. Wiley, New York City, NY, USA.

[23] Corda, C. (2009) Interferometric Detection of Gravitational Waves: The Definitive Test for General Relativity. International Journal of Modern Physics D, 18, 2275-2282. https://arxiv.org/abs/0905.2502 https://doi.org/10.1142/S0218271809015904

[24] Piattella, O.F. (2018) Lecture Notes in Cosmology. https://arxiv.org/pdf/1803.00070.pdf

[25] http://www.tapir.caltech.edu/ teviet/Waves/gwave.html\#: :text=The\%20tidal\%20fi eld\%20g'\%20is, \%E2\%88\%AB\%E2\%88\%AB\%20g'\%20dt\%20\%C2\%B2.

[26] Marek-Crnjac, L. (2011) Quantum Gravity in Cantorian Space-Time. In: Rodrigo Sobreiro, Ed., Quantum Gravity, InTech, Rijeka, Croatia, 97-110.

[27] Vieira, H.S., Bezerra, V.B., Muniz, C.R., Cunha, M.S. and Christiansen, H.R. (2020) Class of Solutions of the Wheeler-DeWitt Equation with Ordering Parameter. Physics Letters B, 809, 135712. https://arxiv.org/abs/2003.13377 https://doi.org/10.1016/j.physletb.2020.135712

[28] Shestakova, T.P. (2019) On the Meaning of the Wave Function of the Universe. International Journal of Modern Physics D, 28, 1941009.

https://arxiv.org/abs/1909.05588 https://doi.org/10.1142/S0218271819410098

[29] Vilenkin, A. (1986) Boundary Conditions in Quantum Cosmology. Physical Review $D, 33,3560$. https://doi.org/10.1103/PhysRevD.33.3560

[30] Arfken, G.G. and Weber, H.J. (1995) Mathematical Methods for Physicists. $4^{\text {th }}$ Edition, Academic Press, New York City, New York, USA. 
[31] Selby, S. (1969) CRC Standard Mathematical Tables. $17^{\text {th }}$ Edition, Chemica Rubber Co., Cleveland, Ohio, USA.

[32] Gong, Y.G. and Hou, S.Q. (2018) The Polarizations of Gravitational Waves. Universe, 4, 85. https://arxiv.org/abs/1806.04027 https://doi.org/10.3390/universe4080085

[33] Barros, B.J. and Lobo, F.S.N. (2018) Wormhole Geometries Supported by Three-Form Fields. https://arxiv.org/pdf/1806.10488.pdf

[34] Zinn-Justin, J. (2003) Phase Transitions and Renormalization Group: From theory to Numbers. In: Duplantier, B. and Rivaseau, V., Eds., Vacuum Energy, Renormalization: Poincare Seminar 2002, Springer, Basel AG, Berlin, 213-239.

[35] Schneider, P. (2002) Nonarchimedean Functional Analysis. Springer, Berlin, Federal Republic of Germany. https://doi.org/10.1007/978-3-662-04728-6

[36] Beckwith, A. (2020) Using "Enhanced Quantization" to Bound the Cosmological Constant, and Quantum Number n for Production of 100 Relic Mini Black Holes and Concluding with a Proof of a Minimum Time Step. Zeldovich 4. September 11 Section on Dark Matter and Dark Energy.

https://www.researchgate.net/publication/344207091_Using_Enhanced_Quantizati on_to_bound_the_Cosmological_constant_and_quantum_number_n_for_producti on_of_100_relic_mini_black_holes_and_concluding_with_a_proof_of_a_minimum time_step

[37] Freese, K., Rindler-Daller, T., Spolyar, D. and Valluri, M. (2016) Dark Stars: A Review. Reports on Progress in Physics, 79, 066902.

https://iopscience.iop.org/article/10.1088/0034-4885/79/6/066902

https://doi.org/10.1088/0034-4885/79/6/066902 